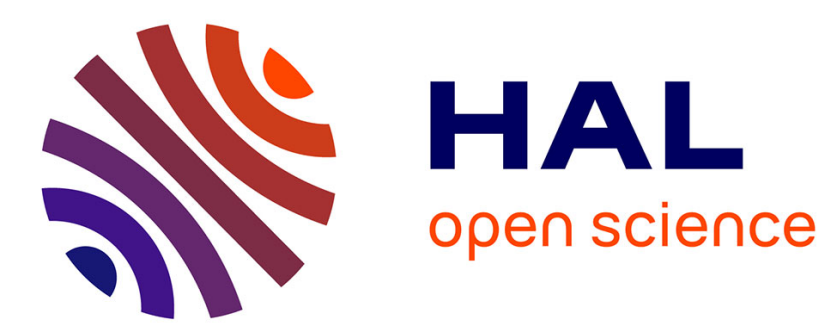

\title{
Drying in a Capped Capillary
}

\author{
Roland Roth, Andrew Parry
}

\section{To cite this version:}

Roland Roth, Andrew Parry. Drying in a Capped Capillary. Molecular Physics, 2011, pp.1. 10.1080/00268976.2011.553638 . hal-00686167

\section{HAL Id: hal-00686167 \\ https://hal.science/hal-00686167}

Submitted on 8 Apr 2012

HAL is a multi-disciplinary open access archive for the deposit and dissemination of scientific research documents, whether they are published or not. The documents may come from teaching and research institutions in France or abroad, or from public or private research centers.
L'archive ouverte pluridisciplinaire HAL, est destinée au dépôt et à la diffusion de documents scientifiques de niveau recherche, publiés ou non, émanant des établissements d'enseignement et de recherche français ou étrangers, des laboratoires publics ou privés. 


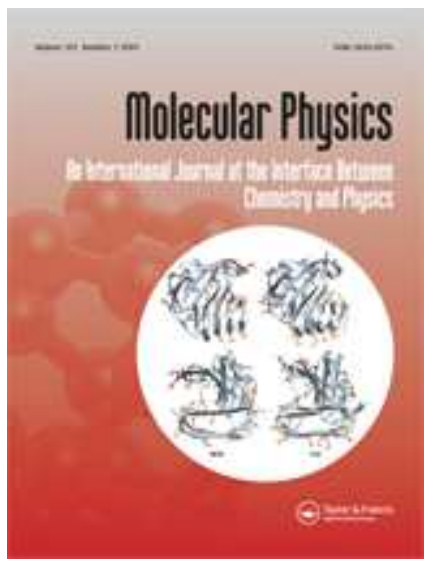

\section{Drying in a Capped Capillary}

\begin{tabular}{|c|c|}
\hline Journal: & Molecular Physics \\
\hline Manuscript ID: & TMPH-2010-0443 \\
\hline Manuscript Type: & Special Issue paper - In honour of Bob Evans \\
\hline $\begin{array}{r}\text { Date Submitted by the } \\
\text { Author: }\end{array}$ & 17-Nov-2010 \\
\hline Complete List of Authors: & $\begin{array}{l}\text { Roth, Roland; University of Erlangen } \\
\text { Parry, Andrew; Imperial College }\end{array}$ \\
\hline Keywords: & $\begin{array}{l}\text { wetting and drying, capillary condensation, density functional } \\
\text { theory }\end{array}$ \\
\hline \multicolumn{2}{|c|}{$\begin{array}{l}\text { Note: The following files were submitted by the author for peer review, but cannot be converted } \\
\text { to PDF. You must view these files (e.g. movies) online. }\end{array}$} \\
\hline source files.zip & \\
\hline
\end{tabular}

\section{SCHOLARONE \\ Manuscripts}


Molecular Physics

Vol. 00, No. 00, Month 200x, 1-13

\title{
RESEARCH ARTICLE
}

\section{Drying in a Capped Capillary}

\author{
Roland Roth ${ }^{a}$ and Andrew O. Parry ${ }^{b}$ \\ ${ }^{a}$ Institut für Theoretische Physik I, Universität Erlangen, Staudtstr. 7, 91058 Erlangen, \\ Germany; ${ }^{b}$ Department of Mathematics, Imperial College London, London SW7 2BZ, UK \\ (Received 00 Month 200x; final version received 00 Month 200x)
}

\begin{abstract}
We use a model Density Functional, based on the White-Bear version of Fundamental Measure theory, to test recent predictions, due to Evans and co-workers, that capillary condensation in a capped capillary-slit is a continuous interfacial critical phenomenal related to the complete wetting transition. Using a model with a square-well intermolecular fluid-fluid attraction we first determine accurately the location of the first-order capillary evaporation transition in an infinite (open) hard-wall capillary slit. Extending the density functional model to allow for a two dimensional order-parameter profile, we then study the adsorption as the chemical potential is reduced to capillary evaporation but now in a capillary-slit that is capped at one end. The equilibrium density profiles obtained show that, sufficiently close to the phase boundary, a meniscus separating liquid-like and vapour-like phases forms near the capped end, and that as capillary evaporation is approached, continuously unbinds from the capped end. Our numerical results indicate that the divergence of the adsorption due to the unbinding of the meniscus is logarithmic and is the same as for the complete wetting transition in systems with short-ranged forces.
\end{abstract}

\section{Introduction}

In 1979 Bob Evans wrote a pioneering review article [1] on the application of classical density functional theory (DFT) to the statistical mechanics of inhomogeneous fluids and, with exceptional clarity, explained how free-energies, density profiles and correlation functions can all be calculated from a model functional. It is no exaggeration to say that this brought "calculation to the masses" paving the way for the development of ever more sophisticated model microscopic functionals [27] together with their application to phase transitions such as wetting, layering and capillary condensation [8-12]. Together with effective interfacial Hamiltonian models and computer simulations, DFT is part of the triumvirate of powerful tools theoreticians have used to revolutionise our understanding of interfacial phenomena, which has grown enormously since the 1980s. Interest in the last decade has been further renewed by technological developments which allow one to pattern and sculpt solid surfaces on the nanometre and micrometre scale. Thus as well as considering adsorption at flat substrates and in capillary slits and pores one can consider corrugated surfaces and wedges/grooves of different cross-section [1317], and also heterogeneous surfaces patterned into domains with different wetting properties. As well as being of huge practical importance this is of fundamental interest to statistical mechanics since different substrate geometry can induce novel types of interfacial phase transition which lie "between" wetting and capillarycondensation. For example there has been a great deal of interest in the physics of filling transitions for fluids adsorbed in linear wedges which can exhibit very large interfacial fluctuation effects compared to wetting at flat substrates $[18,19]$. It has also been noticed, from studies based primarily on effective interfacial Hamilto-

ISSN: 0040-5167 print/ISSN 1754-2278 online

(C) 200x Taylor \& Francis

DOI: $10.1080 / 0040516 Y Y x x x x x x x x$

http://www.informaworld.com 
nian models, that there appear to be precise connections between absorptions in different geometries and between apparently different phase transitions [20, 21]. The purpose of the present article is to test some of these predictions using more microscopic density functional models. By doing so we follow in spirit an approach pioneered by Bob Evans.

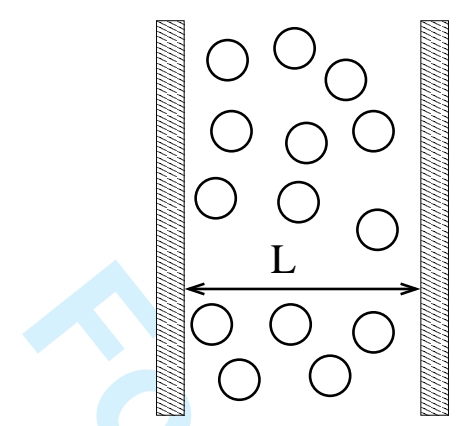

(a)

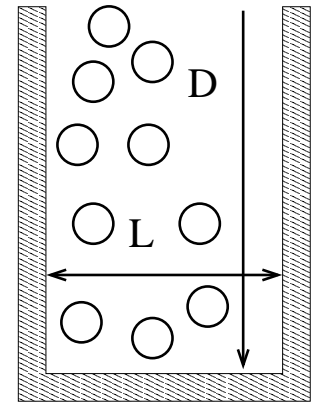

(b)

Figure 1. The geometry of an infinite slit (a) of two parallel hard walls which are separated by the width $L$ and of a capped capillary (b) with width $L$. In case (b) the length $D$ of the capillary is assumed to be infinite.

Capillary-condensation refers to the shift of the liquid-vapour coexistence curve when a fluid is confined in a capillary slit or pore. Thus vapour confined in a slit of width $L$, as shown in Fig. 1(a), will condense to a liquid-like phase, at a pressure $p_{C}(L, T)$, which is different to the value $p_{\text {sat }}(T)$ at bulk coexistence, given by the macroscopic Kelvin equation

$$
p_{\text {sat }}(T)-p_{C}(T, L)=\frac{2 \sigma \cos \theta}{L}+\cdots
$$

where $\sigma$ is the liquid-vapour surface tension and $\theta$ is the contact angle. Thus $p_{C}(L, T)<p_{\text {sat }}(T)$ for contacts angles $0 \leq \theta<\pi / 2$ corresponding to capillary condensation, while $p_{C}(L, T)>p_{\text {sat }}(T)$ for contact angles $\pi / 2<\theta \leq \pi$ corresponding to capillary evaporation. The vast majority of studies of capillary condensation based on Landau-like square gradient models and density-functional theories, assume that the walls are of infinite area and the density is translationally invariant parallel to them. Thus the equilibrium one-body density profile $\rho(\mathbf{r})=\rho(x)$ where $x$ is the co-ordinate perpendicular to the wall(s). In this case capillary condensation is certainly a first-order phase transitions and mean-field treatments of it display adsorption isotherms with a van der Waals loop. However capping the capillary at one end [see Fig. 1(b)] may have a profound effect on this transition. Building on earlier numerical studies, Parry, Rascon, Wilding and Evans [22], pointed out that the presence of the capped end necessarily induces the formation of a meniscus, separating liquid-like and vapour-like phases. For walls that are completely wet by liquid interfacial Hamiltonian models indicate that the meniscus is bound near to the capped end for pressures $p<p_{C}(T, L)$. As the pressure is increased to $p_{C}(T, L)$ the meniscus continuously unbinds from the capped end thus continuously filling the capillary with liquid and is directly analogous to the complete wetting transition occurring at flat walls. However instead of the unbinding of a liquid-vapour interface as bulk coexistence is approached, the unbinding is that of a meniscus as the shifted phase boundary (corresponding to capillary condensation) is approached. Thus condensation in a capped capillary is actually a second-order interfacial phase transition. For systems with short-ranged forces, and in the ab- 
sence of fluctuation effects, this implies that the adsorption in the capped capillary should diverge as $\Gamma \approx-\ln \left|p-p_{C}(T, L)\right|$.

We wish to test this prediction with a microscopic DFT model. This is a nontrivial numerical task of course because the equilibrium density profile $\rho(\mathbf{r})=$ $\rho(x, z)$ is no longer translationally invariant (parallel to the walls) and depends explicitly on $z$ the distance from the capped end. We choose a model fluid with a square-well intermolecular potential and a capillary-slit with purely hard walls. These are completely wet by the vapour phase so our attention is on the capillary evaporation which occurs as the pressure is reduced to $p_{C}(T, L)$. In this case the meniscus separates vapour (towards the capped end) from liquid but otherwise the phenomenology is identical. We begin with a brief recap of the density functional method before accurately determining the location of the capillary-evaporation pressure in an infinite slit with open ends (no cap). This precision is necessary in order to test the logarithmic growth predicted for the capped system. Our numerical results for the two dimensional DFT corresponding to the capped capillary are then presented and discussed.

\section{Density Functional Theory}

Within the framework of density functional theory for classical systems $[1,23]$ there exists the grand canonical potential functional of the form

$$
\Omega[\rho]=\mathcal{F}[\rho]+\int d^{3} r \rho(\mathbf{r})\left(V_{\text {ext }}(\mathbf{r})-\mu\right),
$$

where $\mathcal{F}[\rho]$ is the functional of the intrinsic Helmholtz free energy, $V_{\text {ext }}(\mathbf{r})$ is the external and $\mu$ the chemical potential. It can be proven that the functional $\Omega[\rho]$ possesses the property that it is minimal for the equilibrium density distribution $\rho_{o}(\mathbf{r})$, for which it reduces to the grand potential $\Omega=\Omega\left[\rho_{0}\right]$ of the system. It follows that the equilibrium density distribution can be obtained from the variational principle

$$
\left.\frac{\delta \Omega[\rho]}{\delta \rho(\mathbf{r}))}\right|_{\rho(\mathbf{r})=\rho_{0}(\mathbf{r})}=0 .
$$

Once $\rho_{0}(\mathbf{r})$, i.e. the inhomogeneous structure, is known the thermodynamics is accessible through the grand potential $\Omega$.

The functional of the intrinsic Helmholtz free energy

$$
\mathcal{F}[\rho]=\mathcal{F}_{i d}[\rho]+\mathcal{F}_{\text {ex }}[\rho]
$$

can be split into an exactly known ideal gas part $\mathcal{F}_{i d}[\rho]$ and an excess (over the ideal gas) free energy $\mathcal{F}_{\text {ex }}[\rho]$, which for most systems of interest is known only approximately. The excess free energy contains all the information about the inter-particle interactions. Here we wish to study a simple fluid with short-ranged interactions, that can phase separate into a liquid and a vapour phase. A well studied case is the square-well fluid $[24,25]$ with the inter-particle interaction given by

$$
\beta V_{s w}(r)=\left\{\begin{array}{cl}
\infty & r<2 R \\
-\beta \varepsilon & 2 R<r<2 R_{s w} \\
0 & \text { otherwise }
\end{array}\right.
$$


As usual $\beta=1 /\left(k_{B} T\right)$, with the Boltzmann constant $k_{B}$ and the absolute temperature $T$. Within DFT we treat the square-well interaction by a hard-sphere reference system, described by the White-Bear version [5-7] of fundamental-measure theory $[4,26]$, and a square-well attraction as a perturbation:

$$
\mathcal{F}_{\text {ex }}[\rho]=\mathcal{F}_{e x}^{H S}[\rho]+\frac{1}{2} \int d^{3} r \rho(\mathbf{r}) \int d^{3} r^{\prime} \rho\left(\mathbf{r}^{\prime}\right) \phi_{s w}\left(\left|\mathbf{r}-\mathbf{r}^{\prime}\right|\right) .
$$

The perturbation term of this form underestimates the correlation in the system. To compensate this effect, one usually introduces an empirical modified square-well potential

$$
\beta \phi_{s w}(r)=\left\{\begin{array}{cl}
-\beta \varepsilon & r<2 R_{s w} \\
0 & \text { otherwise }
\end{array}\right.
$$

where the attraction is extended into the core, i.e. to $r \rightarrow 0$.

In the absence of any external field, $V_{\text {ext }}(\mathbf{r})=0$, the density profile of the fluid reduces to the constant bulk density $\rho(\mathbf{r})=\rho$ and the density functional provides simple expressions for the chemical potential $\mu(\rho, T)$ and the pressure $p(\rho, T)$ of the fluid. It is straightforward to construct the fluid phase diagram by demanding mechanical- and chemical-equilibrium between two fluid phases with densities $\rho_{I}$ and $\rho_{I I}$, respectively, i.e.

$$
\mu\left(\rho_{I}, T\right)=\mu\left(\rho_{I I}, T\right) \quad \text { and } \quad p\left(\rho_{I}, T\right)=p\left(\rho_{I I}, T\right) .
$$

In the following we chose the range of the square-well attraction to be $R_{s w}=3 \sigma$, where $\sigma$ is the hard-sphere diameter. The resulting phase diagram of a square-well bulk fluid is shown in Fig. 2 in the (dimensionless) density-temperature representation, where $\eta=\rho(4 \pi / 3) R^{3}$ is the packing fraction of the bulk fluid. Note that for a confined fluid, as studied in the following the packing fraction is a measure for the density in the particle reservoir. The DFT calculations presented here are performed at a fixed temperature, corresponding to $\beta \varepsilon=1.1$, as indicated by the dotted line in Fig. 2. The dashed line in Fig. 2 denotes the Fisher-Widom line [27], which separates region in the phase diagram in which the bulk correlation function decays monotonic from those in which it decays oscillatory. Within the framework of DFT Bob Evans and co-workers could show [28-32] that the character of the bulk correlations, i.e. monotonic or oscillatory decay, is also reflected by inhomogeneous density distributions. It is this insight that made us calculate and plot the Fisher-Widom line for our system in Fig. 2. The fluid state points we consider are clearly on the oscillatory side of the Fisher-Widom line.

Before studying the square-well fluid confined by a capped capillary, for which the density profile $\rho(\mathbf{r})=\rho(x, z)$ depends on two coordinates, it is of great importance to establish the accuracy and internal consistency of the implementation of the DFT in order to ensure that the numerical results are reliable. For our study we require consistency between the structure of the fluid, i.e. the density profile $\rho(\mathbf{r})$, and thermodynamic quantities that can be derived from the grand potential $\Omega=\Omega[\rho(\mathbf{r})]$. To this end we verify that our numerical results at a single planar hard wall satisfy the contact theorem and Gibbs' adsorption theorem. Note that we have employed the two-dimensional DFT implementation even at a single planar wall, although the problem could be reduced to an effectively one-dimensional problem. Furthermore, we have verified that our implementation satisfies Gibbs' adsorption theorem in a slit geometry and in a capped capillary. Using the two dimensional implementation with 10 grid points per hard-sphere radius we have established at 
Figure 2. Bulk phase diagram of a square-well fluid with well depth $\varepsilon$ and square-well range $R_{s w}=3 \sigma$. The dashed line is the Fisher-Widom line that separates the phase diagram into a region in which the bulk correlation function decays monotonic and one in which it decays oscillatory. We perform our DFT calculations at a fixed temperature, corresponding to $\beta \varepsilon=1.1$, as indicated by the dotted line, i.e. in the oscillatory region of the phase diagram. For this temperature and the employed slit width, $L=19.6 R$, capillary evaporation occurs at $\eta_{C E}(T, L)=0.360641$ (marked by the circle), well away from bulk phase coexistence. The full line indicates the path in the phase diagram taken for our calculations in the capped capillary.

the fixed temperature shown in Fig. 2 for various densities that the relative error of the sum rules is less than $10^{-5}$.

\section{Infinite Slit}

It is well known and studied that for all values of $T$ between the critical temperature $T_{c}$ and the triple point temperature $T_{t}$ at a single planar hard wall complete drying occurs for a square-well liquid as bulk coexistence with the vapour phase is approached, i.e. as the chemical potential $\mu \rightarrow \mu_{\text {sat }}(T)$ approaches from above its value at coexistence, $\mu_{\text {sat }}(T)$, a macroscopically thick vapour film wets the interface between the bulk liquid and the planar hard wall. It is also well established that a square-well liquid off coexistence, $\mu>\mu_{\text {sat }}(T)$, confined by two parallel planar hard wall (slit) at separation $L$ undergoes the phase transition of capillary evaporation $(C E)$ as the chemical potential $\mu \rightarrow \mu_{C E}(T, L)$ approaches the value $\mu_{C E}(T, L)>\mu_{\text {sat }}(T)$ from above [12]. At $\mu=\mu_{C E}(T, L)$ a high density liquid and a low density vapour, that is meta-stable in the bulk, can coexist in a slit of width $L$.

The slit enters our DFT calculation via the external field

$$
\beta V_{\text {ext }}^{\text {slit }}(x)= \begin{cases}\infty & |x|>\frac{L}{2} \\ 0 & \text { otherwise }\end{cases}
$$

In order to determine the location of the capillary evaporation we perform a series of DFT calculations for a fixed temperature, which corresponds to a dimensionless square-well depth of $\beta \varepsilon=1.1$, as indicated by the dotted line in Fig. 2. For practical reasons we fix the slit width to $L=19.6 R$ and vary the chemical potential, or equivalently $\eta$ the reservoir packing fraction of the liquid. For values of 
$\mu>\mu_{C E}(T, L)$ we observe a high density liquid in the slit, while for $\mu<\mu_{C E}(T, L)$ the density profile jumps from a high density liquid profile to a low density vapour one [12]. However, the jump in the density profile is not sufficient to locate the capillary evaporation phase transition, because of hysteresis effects [12]. Within DFT it is possible to calculate the grand potential of the system in addition to the density profile. In Fig. 3 we show the grand potential $\Omega=\Omega[\rho(x)]$ as a function of the reservoir packing fraction obtained from DFT for a square-well fluid in a slit with fixed width. As can be seen, there are two distinct branches, one corresponding to a liquid (squares, full line) and one corresponding to a vapour (circles, dashed line). The grand potential of the liquid depends much stronger on the reservoir packing fraction than that of the vapour. The two branches of the grand potential intersect at the capillary evaporation phase transition, which can be described by $\eta_{C E}(T, L)$ or equivalently $\mu_{C E}(T, L)$. We find for our choice of parameters the capillary evaporation to correspond to a reservoir packing fraction of $\eta_{C E}(T, L)=0.360641$, which is marked by the circle in Fig. 2 .

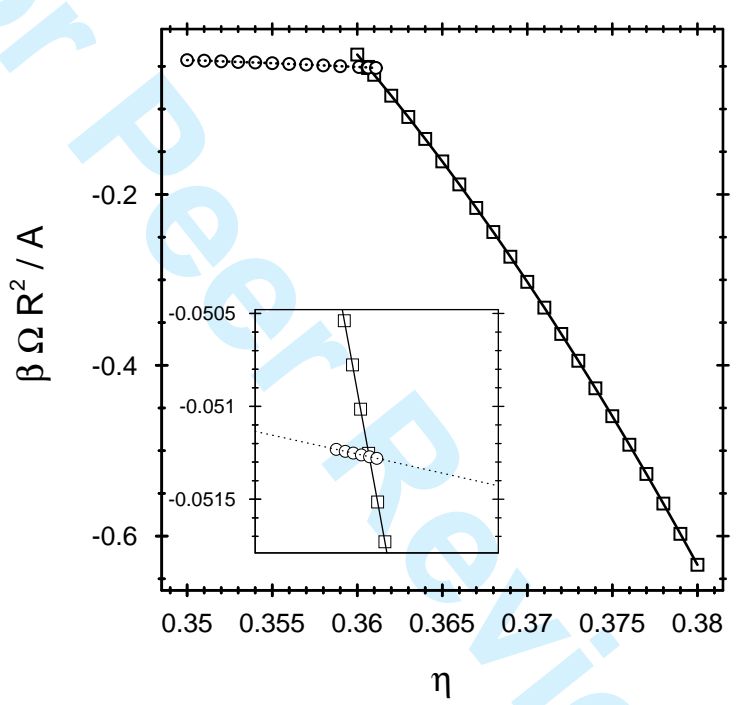

Figure 3. Grand potential per unit area $\Omega$ of a square-well fluid in an infinite slit. The temperature is chosen so that $\beta \varepsilon=1.1$ (see dotted line in Fig. 2). The grand potential of the liquid branch (squares) and that of the vapour branch (circles) intersect at the point of capillary evaporation, corresponding to a reservoir packing fraction of $\eta_{C E}(T, L)=0.360641$ (circle in Fig. 2).

In the inset of Fig. 3 the grand potential of both the liquid and the vapour branch is shown in the vicinity of capillary evaporation. It is clear that there are hysteresis effects and meta-stable liquids or vapours can be observed in the slit, as is typical for a first order phase transition. Note that the accurate location of capillary evaporation can only be determined, if the theory describes the structure (density profiles) and thermodynamics (grand potential) of the system consistently. It is for this reason that we have verified that the contact theorem at a single wall and the Gibbs adsorption theorem at a single wall and in the slit are satisfied.

For a reservoir packing fraction of $\eta=\eta_{C E}(T, L)=0.360641$ a liquid and a vapour can coexist in the slit for the given parameters, $L=19.6$ and $\beta \varepsilon=1.1$. We plot he corresponding density profiles of the coexisting phases in Fig. 4. The density profile of the liquid possesses an oscillatory high density part in the middle of the slit, reflecting the fact that its state point is located in the oscillatory region of the phase diagram (see Fig. 2), and has a contact value at the walls, $|x|=L / 2$, which is significantly lower than the reservoir density [24]. 


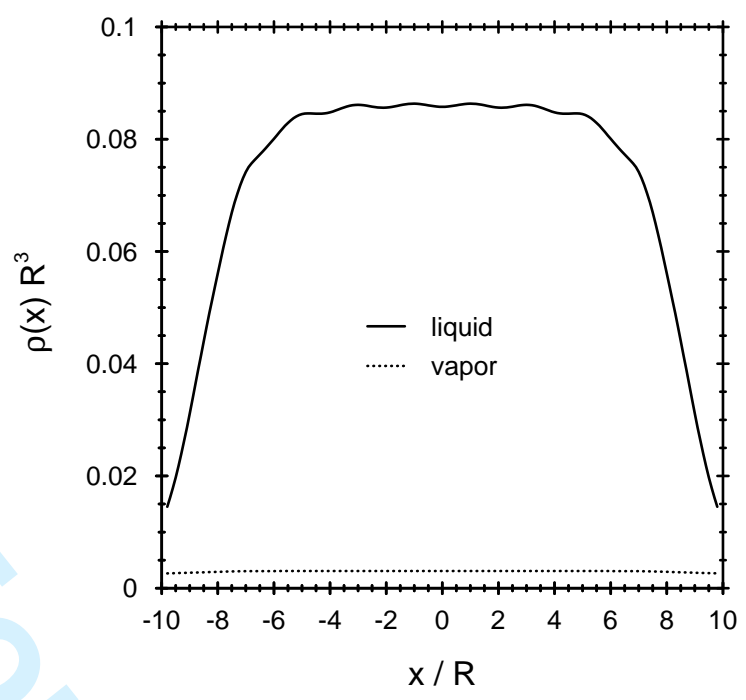

Figure 4. Density profiles of a square-well fluid in an infinite slit at the coexisting reservoir packing fraction of $\eta=\eta_{C E}(T, L)=0.360641$. The difference between the liquid and the vapour profile is well pronounced. Note that the state point of the high density liquid lies on the oscillatory side of the Fisher-Widom line, while that of the low density vapour is located on the monotonic side. In agreement with this observation we find an oscillatory structure only in the liquid profile. As the capillary evaporation is crossed the stable state of the fluid in the slit jumps between these two profiles.

Since we have chosen a temperature well below the critical temperature the difference between the high density liquid and the low density gas at the same chemical potential is large. As a consequence, the change in the density profile as the point of capillary evaporation is crossed is big.

\section{Capped Capillary}

In order to study the behaviour of the square-well fluid in a capped capillary we change the external potential from the slit potential, Eq. (9), to that of a capped capillary

$$
\beta V_{e x t}^{c a p}(x, z)= \begin{cases}\infty & |x|>\frac{L}{2} \text { or } z<0 \\ 0 & \text { otherwise. }\end{cases}
$$

The geometry of the capped capillary is shown in Fig. 1(b). The length of the capillary, $D$ is assumed to be infinite. Clearly, the resulting density profiles are now functions of $x$ and $z$. The reservoir packing fractions considered here correspond to chemical potentials that are larger than its value at capillary evaporation, i.e. $\mu=\mu_{C E}(T, L)+\delta \mu$ with $\delta \mu>0$. This choice is denoted by the full line at fixed temperature in Fig. 2, which ends at the location of capillary evaporation, marked by the circle.

A typical example of the two dimensional density profile of a square well fluid with a reservoir packing fraction of $\eta=0.38$ in the capped capillary is shown in Fig. 5. The chemical potential for the liquid at $\eta=0.38$ is clearly away from capillary evaporation, as is measured by $\beta \delta \mu=0.3719$.

For $z \rightarrow \infty$, the profile recovers that of an infinite slit with a low contact value at the walls, $|x|=L / 2$, and an oscillatory high liquid density part in the middle of the capillary. The oscillations represent packing effects in the liquid.

For $z \rightarrow 0$ the influence of the third wall becomes apparent. The density in the 


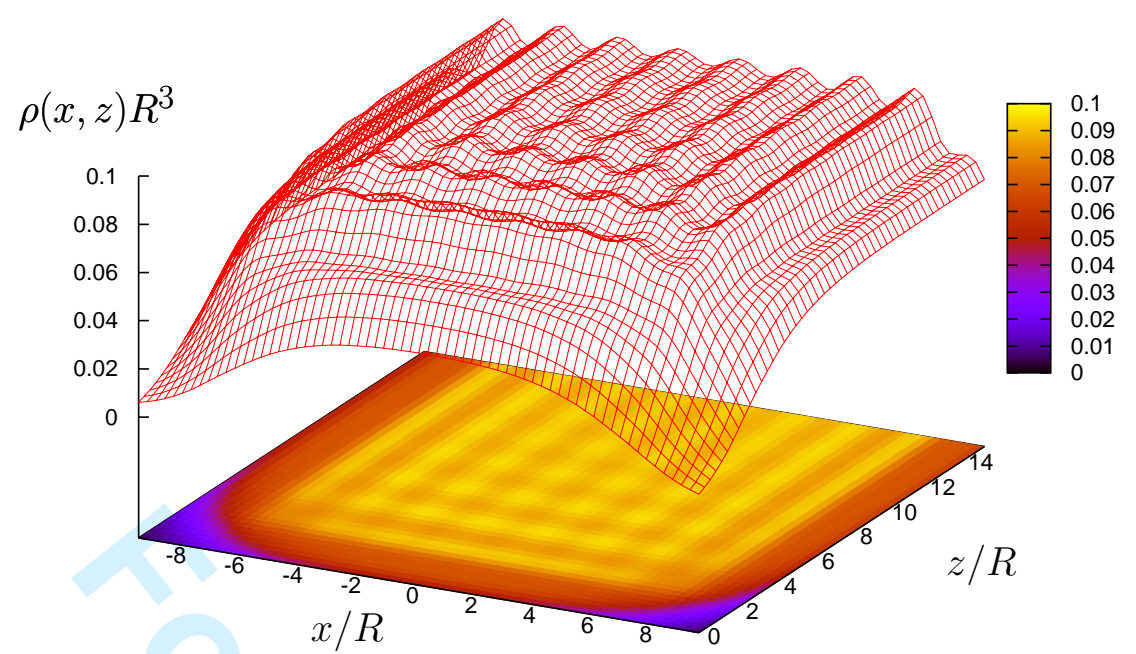

Figure 5. Density profile of a square-well fluid in a capped capillary. The packing fraction is $\eta=0.380$. Corresponding to this packing fraction $\beta \delta \mu=0.3719$.

wedges at $z=0$ and $|x|=L / 2$ is the smallest, as expected, but for the density used for Fig. 5 the wedges are still separated by a region along the third wall at $z=0$ with higher density.

As we decrease the reservoir packing fraction $\eta$ or the corresponding distance in chemical potential $\delta \mu$ from capillary evaporation, the density profile $\rho(x, z)$ changes considerably. The first important change takes place at the third wall at $z=0$. The low density regions at the wedges at $|x|=L / 2$ connect to each other, as can be seen in Fig. 6(a)-(b). At the same time the oscillatory structure in the middle of the slit for $z \rightarrow \infty$ becomes weaker. Note that also the contact value of the liquid at the walls at $|x|=L / 2$ for $z \rightarrow \infty$ decreases, but not nearly as fast as the contact value at $z=0$ and $x=0$. In Fig. $6(\mathrm{a})$ and (b) we show density profiles for reservoir packing fractions of $\eta=0.375$ and 0.370 , which corresponds to $\beta \delta \mu=0.2659$ and 0.1670 , respectively.

As we further approach the location of capillary evaporation, one can observe that a vapour film wets the third wall at $z=0$ [22], as is shown in Fig. 6(c) and (d), for reservoir packing fractions of $\eta=0.365$ and 0.36067 . The corresponding values of $\beta \delta \mu$ are 0.0749 and 0.00049 , respectively.

While it is very interesting to get an impression of the behaviour of the density profile as capillary evaporation is approached, it is important to quantify this phenomena by thermodynamic quantities. Here we consider

$$
\Omega_{e x}=\Omega[\rho(x, z)]+p V
$$

the excess (over a bulk liquid) grand potential. The grand potential of the corresponding bulk system is simply $\Omega_{b u l k}=-p V$, which assumes as a reference system a liquid with constant density that occupies a volume $V$. Note that while the total grand potential of the system is well defined and unique, the excess grand potential is not because the volume $V$ is not uniquely defined. Here we use the volume that is accessible to the centrers of fluid spheres.

Strictly speaking, the thermodynamic limit of Eq. (11), in which the system size becomes infinite, is problematic because both $\Omega$ and $\Omega_{e x}$ are infinite in this limit. Since we are using a fixed, and finite system size in our DFT calculations, we do not face this problem in practice. The finite system considered here is extended by the behaviour of the liquid in an infinite slit, i.e. we make use of the fact that 
1

2

3

4

5

6

7

8

9

10

11

12

13

14

15

16

17

18

19

20

21

22

23

24

25

26

27

28

29

30

31

32

33

34

35

36

37

38

39

40

41

42

43

44

45

46

47

48

49

50

51

52

53

54

55

56

57

58

59

60
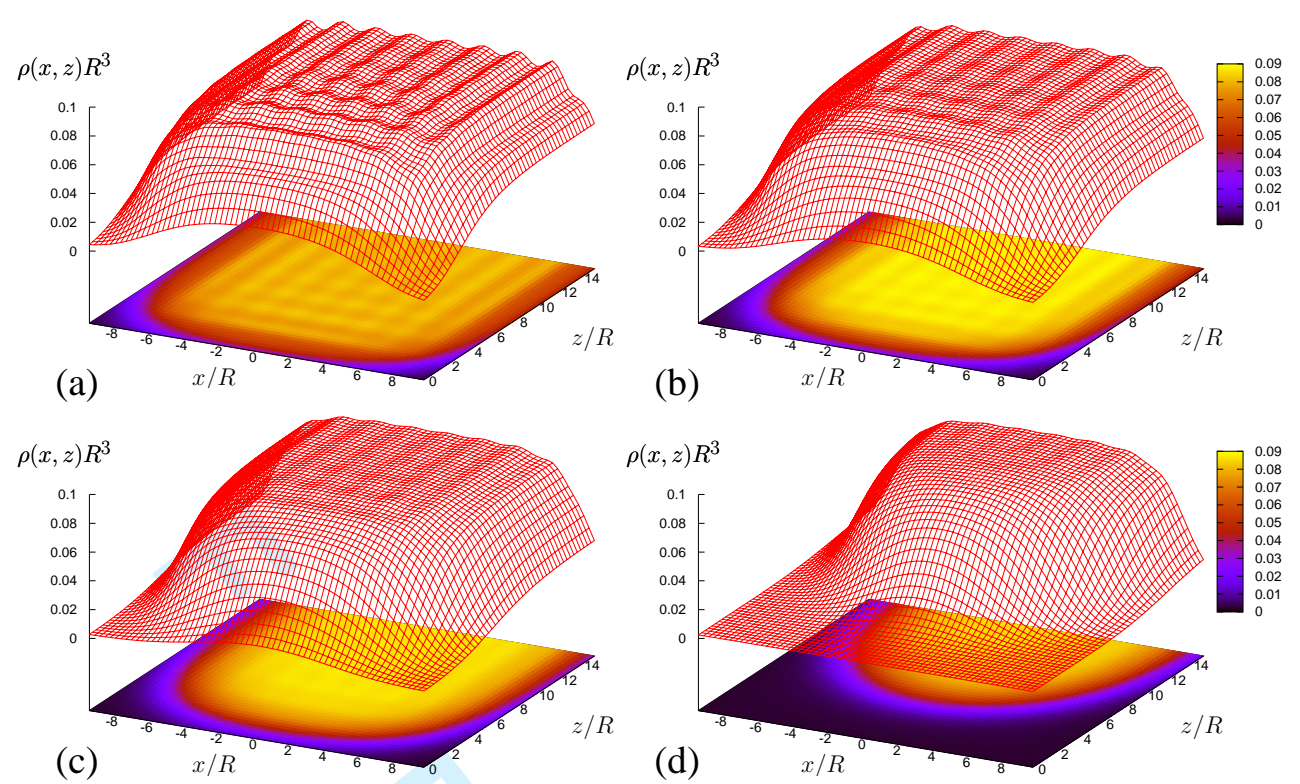

Figure 6. Density profiles of a square-well fluid in a capped capillary. The packing fractions are (a) $\eta=$ 0.375 , (b) 0.370 , (c) 0.365 , and (d) 0.36067 , respectively. These values of $\eta$ correspond to (a) $\beta \delta \mu=0.2659$, (b) 0.1670, (c) 0.0749 , and (d) 0.00049 , respectively.

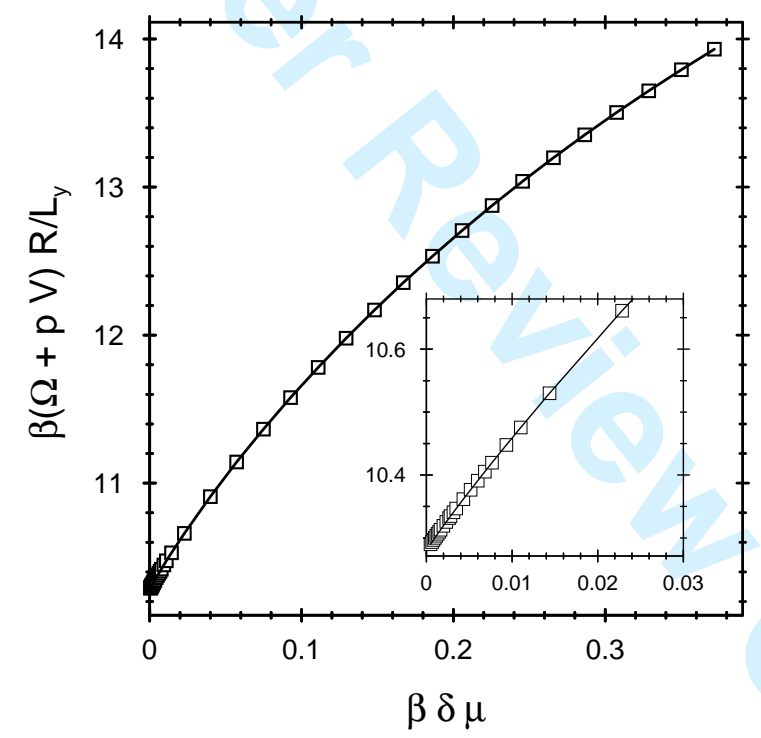

Figure 7. The excess Grand potential $\Omega+p V$ of a square-well fluid in a capped capillary as a function of $\delta \mu=\mu-\mu_{C E}$. The line is a guide to the eye.

$\rho(x, z \rightarrow \infty) \rightarrow \rho^{s l i t}(x)$, where $\rho^{\text {slit }}(x)$ is the density profile of the liquid at the same state point in a slit geometry. We have chosen the system size sufficiently large that this asymptotic behaviour is realised.

In order to isolate the effects of the third wall at $z=0$ it would be cleaner to subtract from $\Omega$ the grand potential of a liquid confined by a slit of volume $V$, rather than that of a bulk liquid. However, the influence of the parallel walls for $z \rightarrow \infty$ is rather small and for the following discussion of no importance so that we can stick to the definition of the excess grand potential given in Eq. (11). 
Figure 8. The excess adsorption $\Gamma$ of a square-well fluid in a capped capillary as a function of $\delta \mu=\mu-\mu_{C E}$. The line is a guide to the eye. $\Gamma$ diverges for $\delta \mu \rightarrow 0$ like $\ln \delta \mu$.

Related to the excess grand potential is the excess adsorption defined by

$$
\Gamma=\int_{V} d^{3} r(\rho(\mathbf{r})-\rho)=-\left.\left(\frac{\partial \Omega_{e x}}{\partial \mu}\right)\right|_{T, V}
$$

which quantifies the excess (over a bulk system) amount of liquid found in the capped capillary. As indicated in Eq. (12) the excess adsorption can be either calculated from the inhomogeneous density profile $\rho(x, z)$, or using Gibbs' adsorption theorem as a derivative of the excess grand potential, Eq. (11), w.r.t. the chemical potential $\mu$. We have verified that for our results both routes are equivalent.

Since the excess grand potential and the excess adsorption are related via Gibbs' adsorption theorem, the same remarks about the thermodynamic limit and the reference system apply.

We show the DFT results for $\Omega_{e x}$ as a function of $\delta \mu$ in Fig. 7. The excess grand potential of the liquid in a capped capillary decreases as the limit $\delta \mu \rightarrow 0$ is approached. In the inset of Fig. 7 we show $\Omega_{e x}$ for very small values of $\delta \mu$. In the whole range we considered the excess grand potential is well behaved.

The situation is clearly different for the excess adsorption, shown in Fig. 8. $\Gamma$ is negative and decreases in the limit $\delta \mu \rightarrow 0$, however, in contrast to the excess grand potential, the decrease in $\Gamma$ becomes stronger with smaller values of $\delta \mu$. In fact for $\delta \mu \rightarrow 0$ one can observe a divergence of the excess adsorption. This is highlighted in the inset of Fig. 8. In order to better understand the divergence we fit the DFT data. Clearly, a polynomial in $\delta \mu$ is not sufficient to capture the behaviour of $\Gamma$ in the range of $\delta \mu$ shown in Fig. 8. However, we find, in accordance with the prediction by Rascon and Parry [13, 22], that the excess adsorption can very nicely be fitted by a polynomial in $\delta \mu$ plus a logarithmic term, i.e. in the limit $\delta \mu \rightarrow 0$ we find that $\Gamma \propto-\ln \delta \mu$. The corresponding term in the excess grand potential in the same limit is given by $\Omega_{e x} \propto \delta \mu \ln \delta \mu$, which is non-analytic but remains finite.

It is also interesting to consider cuts $\rho(x=0, z)$ of the two-dimensional density profiles at the middle of the capped capillary. The predictions of Rascon and Parry 
$[13,22]$ suggest that these cuts should essentially behave like wetting (or drying) profiles of the fluid at a single planar wall, when bulk coexistence is approach as $\delta \mu \rightarrow 0$. Some cuts are shown in Fig. 9 .

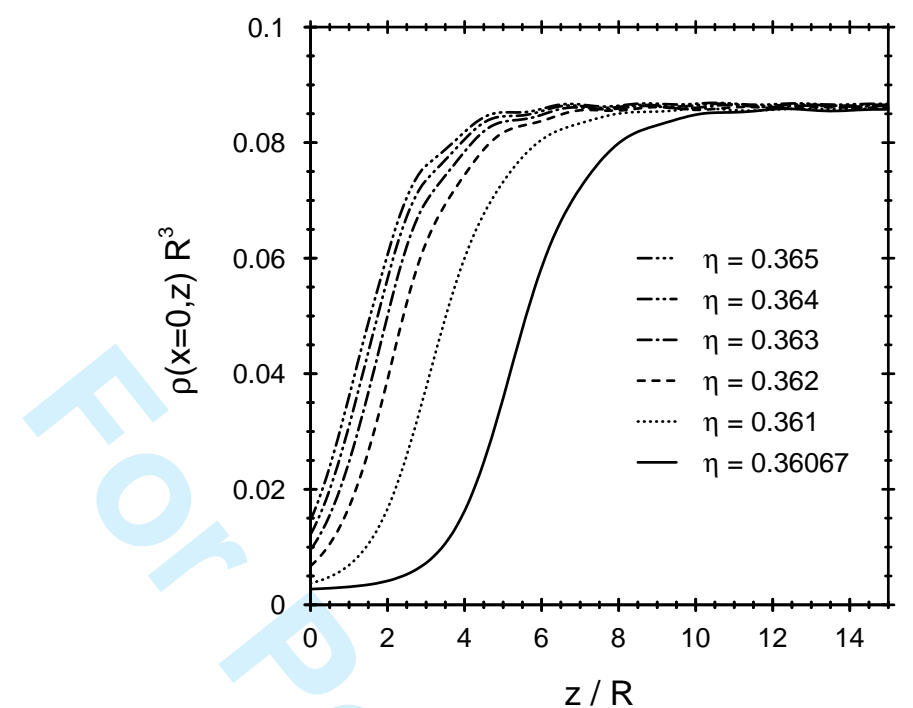

Figure 9. The density $\rho(x=0, z)$ in the middle of the capped capillary, as capillary evaporation is approached with $\delta \mu=\mu-\mu_{C E}(T, L) \rightarrow 0$. These profiles behave essentially like those of a fluid at a single planar wall, as bulk coexistence is approach with $\delta \mu=\mu-\mu_{\text {sat }}(T) \rightarrow 0$.

Due to the close connection between the excess grand potential and the excess adsorption, we can test the consistency of the fits. The fit parameters for the excess adsorption can be used, up to a constant, to describe the data for the excess grand potential, including the non-analytic contribution.

It is important to realise that the fact that the divergence in the excess adsorption is (numerically) observed at $\delta \mu=0$ is a consequence of the high level of consistency between the structure and thermodynamic quantities in our implementation of DFT. Any slight discrepancy could result in an apparent shift of the divergence.

\section{Discussion}

In this paper we have used the White-Bear version of fundamental measure DFT to investigate the nature of capillary evaporation in a capped hard-wall capillary slit. Our numerically results support the prediction made using effective interfacial Hamiltonian models predict that in this geometry capillary evaporation is a secondorder transition related to complete wetting. This is perhaps most clearly seen in our final diagram (Fig. 9) which shows various density profiles (measured at the mid-point between the walls) as a function of the distance from the capped end on approaching capillary evaporation. These profiles resemble, qualitatively, those for drying films at a planar wall and show the growth of a thick "drying" layer as "coexistence" is approached. However in this case the "wall" is the capped end, coexistence refers to capillary evaporation, the unbinding "interface" is actually a cross-section of the meniscus, and the coexisting phases are the "liquid-like" and "vapour-like" phases of the shifted bulk coexistence curve. Using an accurate and independently determined value for $\mu_{C E}$, we have shown that the growth and divergence of the thickness of the vapour-like phase (the distance of the meniscus to the cap) as $\delta \mu=\mu-\mu_{C E}$ is $-\ln \delta \mu$ - the same as for complete wetting with 
short-ranged forces in accordance with the predictions of Evans and co-workers.

Our present model DFT calculation is of course only mean-field like and does not include interfacial fluctuation effects. For the present case of systems with shortranged forces these could be large and eventually lead to cross-over behaviour in which the adsorption changes from its mean-field expression $\Gamma \propto-\ln \delta \mu$ to $\Gamma \propto \delta \mu^{-1 / 3}$. The latter is the universal result for complete wetting (in bulk two dimensions). Such cross-over behaviour would be very interesting to study as a function of the slit width. Our intuition is that the wider the slit, the narrower the true asymptotic regime and the broader the regime when the mean-field calculation is valid. Unfortunately none of this is as yet possible using DFT and one must still resort to coarse-grained interfacial models and simulation studies.

However there are still many interesting questions one can ask using the present DFT and substrate geometry. For example it would be interesting, and relatively simple, to add an attractive potential to the wall-fluid interaction in order to alter the contact angle. Indeed one may use a different material to cap the capillary so that the interaction potential with the side walls and cap is different. By doing this we might be able to alter the manner in which the meniscus unbinds from the capped end similar to studies of tri-criticality filling transitions in linear wedges. Similarly it would be interesting to see what happens as one moves along the capillary-evaporation line towards the capillary-critical point. In this case we can expect cross-over to critical-adsorption-like behaviour in which the decay of the profile from the capped end follows a slow algebraic power law. Finally we hope that our present work serves to encourage further density functional model studies of adsorption on structured surfaces including more complex fluids and their mixtures.

\section{Acknowledgments}

We are just two of the masses who were taught how to calculate by Bob (although any mistakes in this article are entirely our own). It is our heartfelt pleasure to thank him for his deep physical insight and the inexhaustible encouragement he has always given us.

\section{References}

[1]R. Evans, Adv. Phys. 28, 143 (1979).

[2]P. Tarazona and R. Evans, Mol. Phys. 52, 847 (1984).

[3]P. Tarazon, Phys. Rev. A 31, 2672 (1985).

[4] Y. Rosenfeld, Phys Rev. Lett. 63, 980 (1989).

[5]R. Roth, R. Evans, A. Lang, and G. Kahl, J. Phys.: Condens. Matter 14, 12063 (2002).

[6] Y.-X. Yu and J. Wu, J. Chem. Phys. 117, 10156 (2002).

7] H. Hansen-Goos and R. Roth, J. Phys.: Condens. Matter 18, 8413 (2006).

[8]S. Dietrich, in Phase Transitions and Critical Phenomena, edited by C. Domb and J. L. Lebowitz (Academic, New York, 1988), Vol. 12, p. 1.

[9]R. Evans and P. Tarazona, Phys. Rev. Lett. 52, 557 (1984).

[10]R. Evans, U. Marini Bettolo Marconi, and P. Tarazona, J. Chem. Phys. 84, 2376 (1986).

[11]R. Evans, U. Marini Bettolo Marconi, and P. Tarazona, J. Chem. Soc., Farady Trans. 2 82, 1763 (1986).

[12]R. Evans, J. Phys. Condens. Matter 2, 8989 (1990).

[13] C. Rascon and A. O. Parry, Nature 407, 986 (2000).

[14] O. Gang, K. J. Alvine, M. Fukuto, P. S. Pershan, C. T. Black, and B. M. Ocko, Phys. Rev. Lett. 95, 217801 (2005)

[15] M. Tasinkevych and S. Dietrich, Phys. Rev. Lett. 97, 106102 (2006).

[16]L. Bruschi, G. Fois, G. Mistura, M. Tormen, V. Garbin, E. di Fabrizio, A. Gerardino, and M. Natali, J. Chem. Phys. 125, 144709 (2006)

[17]H. Bohlen, A. O. Parry, E. Diaz-Herrera and M. Schoen, Eur. Phys. J. E 25, 103 (2008).

[18] A. O. Parry, C. Rascon, and A. J. Wood, Phys. Rev. Lett. 85, 345 (2000).

[19]A. Milchev, M. Müller, K. Binder, and D. P. Landau, Phys. Rev. Lett. 90, 136101 (2003).

[20]A. O. Parry, A. J. Wood, and C. Rascon, J. Phys.: Condens. Matter 13, 4591 (2001).

[21]C. Rascon and A. O. Parry, Phys. Rev. Lett. 94, 096103 (2005). 
[22] A. O. Parry, C. Rascon C, N. B. Wilding, and R. Evans, Phys. Rev. Lett. 98, 226101 (2007).

[23]R. Evans, in Fundamentals of In homogeneous Fluids, edited by D. Henderson (Marcel Dekker, New York, 1992) p. 85.

[24]R. Evans, R. Roth, and P. Bryk, Europhys. Lett. 62, 815 (2003).

[25]R. Evans, J. R. Henderson, and R. Roth, J. Chem. Phys. 121, 12074 (2004).

[26]R. Roth, J. Phys.: Condens. Matter 22, 063102 (2010).

[27]M. E. Fisher and B. Widom, J. Chem. Phys. 50, 3756 (1969).

[28]R. Evans, J. R. Henderson, D. C. Hoyle, A. O. Parry, and Z. A. Sabeur, Mol. Phys. 80, 755 (1993).

[29]R. Evans, R. J. F. Leote de Carvalho, J. R. Henderson, and D. C. Hoyle, J. Chem. Phys. 100, 591 (1994).

[30]R. J. F. Leote de Carvalho, R. Evans, D. C. Hoyle, and J. R. Henderson, J. Phys.: Condens. Matter 6, 9275 (1994).

[31]P. Hopkins, A. J. Archer and R. Evans, Phys. Rev. E 71, 027401 (2005).

[32]R. Evans and J. R. Henderson, J. Phys.: Condens. Matter 21, 474220 (2009). 